\title{
Characterising the lean polycystic ovary syndrome (PCOS) population: behavioural, anthropometric and biochemical presentation
}

\author{
K. Hart ${ }^{1}$, S. Barr ${ }^{2}$ and Y. Jeanes ${ }^{2}$ \\ ${ }^{1}$ Faculty of Health and Medical Sciences, University of Surrey, Guildford GU2 7XH, UK and ${ }^{2}$ Health Sciences \\ Research Centre, Roehampton University, London SW15 4JD, UK
}

Polycystic ovary syndrome (PCOS) affects $\leq 10 \%$ of women of reproductive age in the $\mathrm{UK}^{(1)}$. Despite their increased risk of diabetes and $\mathrm{CHD}$, there remains a lack of evidenced-based guidelines for the effective dietary management of this population group. The successful strategies that have been identified focus predominantly on the benefits of weight $\operatorname{loss}^{(2)}$, which is inappropriate for the small but significant minority of patients who are normal or underweight. By characterising the lean PCOS population in terms of their disease presentation and behavioural, anthropometric and biochemical patterns the present study aims to inform the development of more appropriate and hence effective management guidelines.

Data were collected from two sources: first, a postal survey of women with PCOS (wPCOS) recruited via the mailing list of the PCOS charity Verity; second, via an observational study of wPCOS and age- and weight-matched controls. Postal volunteers provided self-report data on symptom profile, weight and height, activity levels and dietary intake. Observational-study volunteers provided blood and urine samples for the analysis of hormone and lipid profiles. Oral glucose tolerance tests (OGTT) were performed and weight, height and body composition measurements taken. Dietary and activity data were collected using 1-week food and activity diaries complemented by step counters.

Of the 186 postal wPCOS with weight data $47 \%$ ( $n$ 88) were normal weight or underweight, reporting a mean of 3.9 (SD 1.4$)$ symptoms compared with 4.6 (SD 1.6) in their overweight or obese counterparts (NS). Menstrual and hair-related symptoms were most commonly reported by lean wPCOS ( $82 \%$ and $75 \%$ respectively) compared with weight and hair problems in overweight wPCOS (93\% and $86 \%$ respectively). Of the eight symptom types discussed only weight and fertility issues were more frequently reported in overweight wPCOS $\left(\chi^{2} 42.8, P<0.001\right.$ and $\chi^{2} 4.2, P=0.03$ respectively) whilst normal or underweight wPCOS were more likely to report menstruation issues $\left(\chi^{2} 4.4, P=0.04\right)$. Forty-five lean women were recruited to the observational study. Compared with their non-PCOS counterparts lean wPCOS ( $n$ 25) had a significantly higher total cholesterol (5.24 (SD 0.99) v. 4.60 (SD 0.71); $t-2.44, P=0.02)$ and $2 \mathrm{~h}$ insulin (post-OGTT; 26.78 (SD 11.6) v. 18.74 (SD 7.49); $t-2.52, P=0.02$ ) than lean controls $(n$ 20). Although mean homeostasis model assessment $(\mathrm{HOMA})$-B (pancreatic $\beta$-cell function) was lower and mean HOMA-insulin resistance higher in lean wPCOS than controls, these differences were not significant. No significant difference in activity level (steps per d) was identified and analysis is ongoing to identify any differences in dietary patterns between subgroups and to further elucidate the relationship between weight, behaviour and disease presentation.

Whilst they may not match the commonly-perceived PCOS phenotype, lean wPCOS report clinically-significant disease-related morbidity $^{(3)}$ and may remain at increased risk of CVD compared with the non-PCOS population, highlighting the need for appropriate management strategies that are not reliant on weight loss to achieve clinical improvement. Further analysis of this comprehensive data-set will allow the elucidation of the complex relationship between weight and PCOS presentation and the development of more sophisticated and patient-centred dietary guidelines.

1. Hart R, Hickey M \& Franks S (2004) Best Pract Res Clin Obstet Gynaecol 18, 671-683.

2. Marsh K \& Brand-Miller J (2005) Br J Nutr 94, 154-165.

3. Herriot AM, Whitcroft S \& Jeanes YM (2007) Ann Nutr Metab 51, Suppl.1, 303. 